\section{Und es liegt doch in den}

U mwelt oder Konstitution - im Streit darum, welche Faktoren das Entstehen von Ekzemen bedingen, weisen die Ergebnisse einer Arbeitsgruppe von den Universitäten Heidelberg und Dresden jetzt auf die dominierende Rolle des Erbguts hin. Die Forscher hatten die Daten des Kinder- und Jugendgesundheitssurveys (KiGGS) analysiert, einer deutschlandweiten Querschnittstudie, an der sich in den Jahren 2003 bis 2006 knapp 18.000 Kinder und Jugendliche bis zum Alter von 17 Jahren beteiligt hatten. Wie ausgeprägt eine auf Allergien positive Familienanamnese mit chronischen Hautausschlägen assoziiert ist, lässt sich an der Odds Ratio (OR) von 1,94 ablesen. Andere Faktoren, wie etwa Infektionen oder Ikterus nach

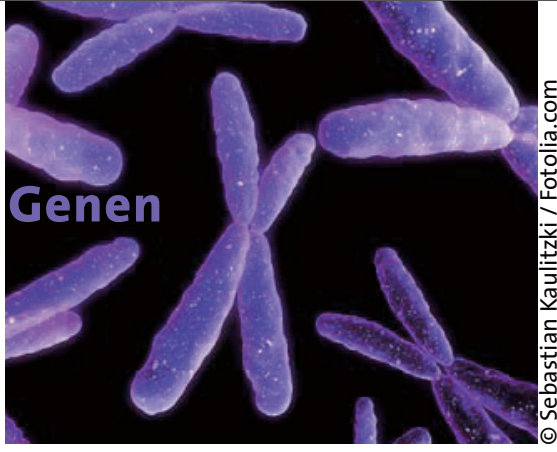

der Geburt, schlugen in puncto Ekzemrisiko deutlich schwächer zu Buche (OR 1,45 bzw. 1,27). Parameter, die Umwelteinflüsse oder Lebensstil abbilden - Alkoholkonsum der Mutter während der Schwangerschaft, Zusammenleben mit Haustieren oder Schimmelbefall der Wohnungswände - hatten keinen signifikanten Einfluss auf die Ekzemhäufigkeit. $\quad r b$

Apfelbacher CJ et al. Allergy 2010 Aug 30 [Epub ahead of print]

\title{
Asthma: Paracetamol als Risikofaktor entlastet
}

aracetamol begünstige bei Kindern und Jugendlichen die Entwicklung von Asthma - darauf deuteten multizentrische Studien eines Teams um Richard Beasley aus Neuseeland hin. Jetzt kommt Widerspruch aus Australien. In einer prospektiven Geburtskohortenstudie wurden bei 495 Kindern mit familiärer Allergiedisposition der Paracetamolverbrauch bis zum zweiten und der Gesundheitszustand bis zum siebten Lebensjahr dokumentiert. Nach Adjustierung der Risikoparameter in Bezug auf die Häufigkeit von Atemwegsinfektionen - die per se als Risikofaktor für Asthma bekannt sind - wurde hier keine Verbindung von früher Paracetamoleinnahme und Asthma gefunden.

Lowe AJ et al. BMJ 2010 Sep 15; 341: C4616. doi: $10.1136 /$ bmj.c4616

\section{Kein Honorar für eine duftende Therapeutin}

E in außergewöhnlicher Rechtsstreit: Im Mai 2008 hatte eine Patientin eine Sitzung bei ihrer Naturheilkunde-Therapeutin abgebrochen. Denn diese hatte ein dezentes Parfüm aufgelegt - obwohl die Patientin gegen Duftstoffe allergisch war. Eben gegen diese Allergie sollte sie behandelt werden. Nun fürchtete die Frau jedoch eine allergische Reaktion wegen des Parfüms. Sie verließ die Praxis und weigerte sich später, die Sitzung zu bezahlen. So geht es nicht, fand die Therapeutin: Sie habe zwar ein Parfüm getragen, dessen Duft sei aber nicht intensiver gewesen als eine Pflege mit einem Deodorant. Das stelle

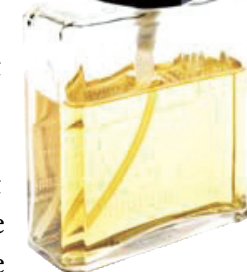

(c) Michałowski Dominik

/ Fotolia.com keinen Grund dar, die Behandlung abzubrechen und das Honorar zu verweigern. Dem widersprach das Amtsgericht Rheinbach. Wer Patienten mit einer Duftallergie behandeln wolle, müsse entsprechende Rücksicht nehmen. Wenn die Therapeutin selbst eine Duftquelle darstelle, verletze sie damit ihre vertragliche Pflicht, die Patientin zu schützen. $\mathrm{Ob}$ ihr Parfüm auffällig gewesen sei oder nicht, spiele dabei keine Rolle: Die bloße Existenz von Duftstoffen könne eine Reaktion hervorrufen.

gri

Amtsgericht Rheinbach vom 19.

Juli 2010 - 5 C 437/o9

\section{Rhinitis mit Todesfolge}

_ ine 43-jährige Frau war in ihrem Auto - unterwegs, als sie eine heftige Niesattacke erlitt und die Kontrolle über ihr Fahrzeug verlor. Dabei stieß sie mit einem anderen Wagen zusammen, dessen Fahrer tödlich verletzt wurde. Die Frau selbst kam leichtverletzt davon. Diese dramatischen Konsequenzen einer allergischen Rhinitis haben jetzt Ärzte aus Los Angeles beschrieben. Bei der Abklärung ihrer Erkrankung acht Monate später berichtete die Frau, seit ihrer Kindheit unter plötzlichen Niesattacken zu leiden. Das geschehe etwa einmal wöchentlich. Eine allergische Genese des Geschehens hatte sie nie in Betracht gezogen, vielmehr glaubte sie, die Anfälle von ihrem Vater „geerbt“ zu haben, der die Symptomatik mit ihr teilte. Hauttests zeigten ausgeprägte Reaktionen auf Graspollen, Sykomoren und Katzen.

$r b$

Spector SL et al. J Allergy Clin Immunol 2010 Aug 30 [Epub ahead of print]

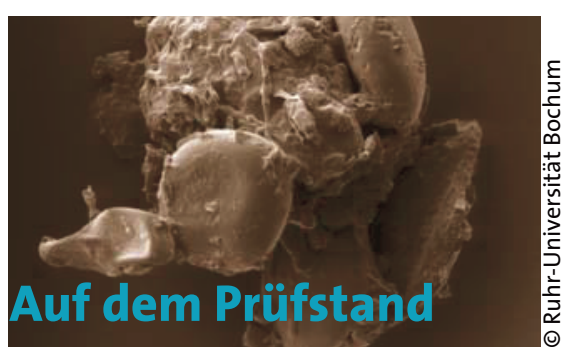

as Polysaccharid Arabinogalaktan soll einer der Wunderstoffe im Stallstaub sein, die vor der Entwicklung allergischer Erkrankungen schützen. Zumindest im Mausmodell funktioniert das sehr gut, wie ein deutsches Forscherteam zeigen konnte. Die dendritischen Zellen der intranasal mit dem Zucker stimulierten Tiere produzierten autokrin Interleukin10 und waren nicht in der Lage, eine allergische Immunantwort zu induzieren. Die Mäuse selbst waren vor atopischer Sensibilisierung, Entzündung und Hyperreaktivität der Atemwege geschützt. Arabinogalaktan kommt in Futterpflanzen wie dem Wiesenfuchsschwanz vor und findet sich auch im Staub von Ställen. $r b$

Peters $\mathbf{M}$ et al. J Allergy Clin Immunol 2010; 126: 648-56 